\title{
Divergent Reaction Mechanisms in the Aminofluorination of Alkenes
}

\author{
Wangqing Kong, Estíbaliz Merino, and Cristina Nevado*
}

\begin{abstract}
The aminofluorination of alkenes has become an attractive platform for the synthesis of $\beta$-aminofluorinated compounds, valuable building blocks in medicinal and agricultural chemistry. The novel methodologies disclosed in recent years have unraveled a broad array of reaction mechanisms, so that the interest in these transformations transcends the mere synthetic aspects. This review aims to summarize the most relevant findings in this area attending at the nature of the fluorine source, and thus the specific mechanism operating in each of these transformations, namely electrophilic, nucleophilic, radical, and late transition metal-catalyzed reactions.
\end{abstract}

Keywords: Alkenes · Aminofluorination · Difunctionalization

\section{Introduction}

The effect of fluorine atoms on the properties of bioactive molecules has been explored in depth. Improvements in solubility, bioavailability and metabolic stability have been thoroughly demonstrated fostering synthetic efforts towards the more efficient introduction of $\mathrm{C}-\mathrm{F}$ bonds in organic building blocks. ${ }^{[1-7]}$ In recent years, the difunctionalization of alkenes has also received increasing attention. ${ }^{[8,9]}$ Dioxygenations, ${ }^{[10-13]}$ aminooxygenations, ${ }^{[14-19]}$ diaminations, ${ }^{\text {[20-24] }}$ and aminohalogenations ${ }^{[25,26]}$ have been described. In addition, the difunctionalization of alkenes with trifluoromethyl groups involving the concomitant formation of $\mathrm{C}-\mathrm{C}$ or $\mathrm{C}$-heteroatom bonds has also been disclosed. ${ }^{[27]}$

In this review, we decided to focus our attention on a particular set of alkene difunctionalizations, namely, those that involve the formal addition of nitrogen and fluorine across the $\pi$ system, and are thus described as alkene aminofluorination processes. Both the intra- and intermolecular version of these transformations provided access, also in a highly stereocontrolled manner, to $\beta$-fluoroamines, which are considered to be relevant building blocks towards the synthesis of heterocycles, peptides and peptidomimetics as well as other bioactive natural products. In this review, representative examples of alkene aminofluorinations have been classified attending at the nature of the fluorine source and hence, the operating mechanism behind each of these transformations, including electrophilic, nucleophilic, radical and metal-mediated reactions, some of which have bloomed in the past five years.

\section{Electrophilic Fluorination}

Back in 1996, Stavber and co-workers reported the first example of an intermolecular alkene aminofluorination using 1-fluoro-4-hydroxy-1,4-diazoniabicyclo[2.2.2] octane bis(tetrafluoroborate) as electrophilic fluorine source and acetonitrile as nucleophile. Fluoroacetamides were obtained in good yields through a fluoro carbocationic intermediate which is attacked by the nitrogen of acetonitrile in a Ritter-type reaction (Scheme 1). ${ }^{[28,29]}$

A broader scope was attained using Selectfluor as $\mathrm{F}^{+}$source in the presence of a catalytic amount of indium(III) fluoride. ${ }^{[30]}$ Various nitriles including propio- and butyronitrile were found to furnish the corresponding $N$-(2-fluoro-1-alkyl) amides in high yields (Scheme 2 ).

A related intramolecular version of these transformations was applied in the synthesis of the fluorinated analogues of Gypsetin and Brevianamide E, two alkaloids that attracted the interest of the synthetic community as promising competitive inhibitors of acylCoA:cholesterol acyltransferase. Taking advantage of FP-T300, an electrophilic fluorinating agent, a fluorination-cyclization sequence was designed so that Fluorobrevianamide E could be obtained from cyclo-L-Trp-L-Pro (Trp $=$ tryptophan, Pro $=$ proline) together with the corresponding syn-bis epimer in a 1:1.6 ratio as shown in Scheme 3.[31]

In an analogous manner, but with allenes as unsaturated precursors, monofluoro pyrrolidines could be prepared in moderate 
yields under mild reaction conditions exclusively using Selectfluor (Scheme 4). ${ }^{[32]}$

Stereocontrolled versions of these transformations were also sought, becoming the subject of intense research efforts. One of the first examples reported the diastereoselective synthesis of $\beta$-amino- $\alpha$-fluoro esters by conjugate addition of lithium (S)-(-)- $N$-benzyl- $N$ $\alpha$-methylbenzylamides onto $t$-butyl cinnamate and ethyl crotonate followed by fluorination with $N$-fluorobenzenesulfonimide (Scheme 5). Lithium coordination to the enolate seemed to be critical for a good stereocontrol as the addition of DMPU disrupted this coordination providing unselective mixtures of products. ${ }^{[33]}$

Organocatalytic tools have been successfully applied in this context. Thus, Brenner-Moyer and co-workers developed a novel organocatalytic cascade reaction, in the first example of an enantioselective intermolecular aminofluorination of $\alpha, \beta$ unsaturated aldehydes. ${ }^{[34]}$ In this process, two different reactions are performed in tandem, both catalyzed by the same proline-derived catalyst shown in Scheme 6. The first step involves the enantioselective conjugate addition of the amine to an in situ generated $\alpha, \beta$-unsaturated iminium intermediate. The resulting (chiral) enamine intermediate is subsequently captured by the electrophilic source of fluorine to produce $\alpha$-fluoro- $\beta$-amino aldehydes in enantiomerically pure form. Interestingly, the products of these reactions could be readily converted into $\alpha$-fluoro- $\beta$-amino acid derivatives with no appreciable loss of enantioselectivity.

Strategies towards enantioselective intramolecular aminofluorination reactions have also been disclosed. Taking advantage of organocatalytic tools, enantioenriched fluorinated $N$-containing tricycles were obtained by fluorocyclization of prochiral indole precursors by Gouverneur and co-workers. ${ }^{[35]}$ The fluorine substituent was installed on a benzylic quaternary stereogenic carbon center yielding tetrahydrofluoroindoles as single diastereoisomers (d.r.>20:1) (Scheme 7). Selectfluor in combination with a stoichiometric amount of (DHQ) ${ }_{2}$ PHAL, or NFSI with a catalytic amount of the chiral ligand offered comparable results with enantioselectivities up to $92 \%$ ee .

An alternative approach reported by Toste's group relies on the use of chiral phosphoric acids and Selectfluor under anionic phase-transfer catalysis conditions. An enantioselective 1,4-fluoroamination of conjugated dienes could be designed that proceeded through a highly regioselective 6-endo-trig cyclization that afforded benzo[f]isoquinoline derivatives in up to $96 \%$ ee (Scheme 8). ${ }^{[36]}$

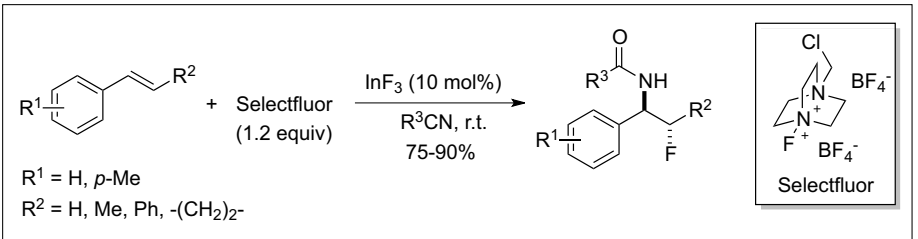

Scheme 2. $\mathrm{InF}_{3}-$ catalyzed fluoroamidation of vinyl arenes.

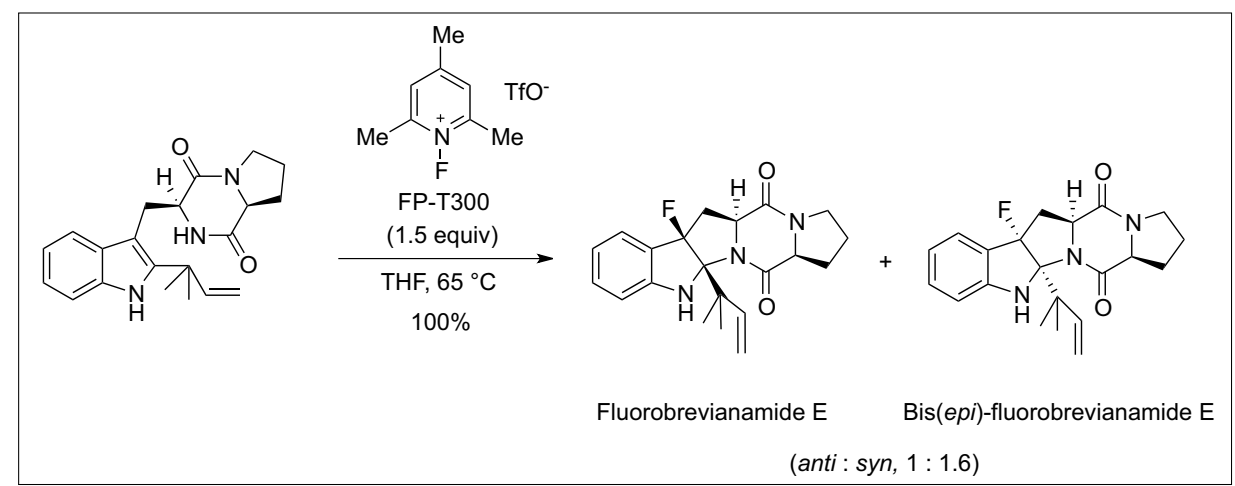

Scheme 3. Synthesis of Fluorogypsetin and Fluorobrevianamide E by fluorination-cyclization reaction.

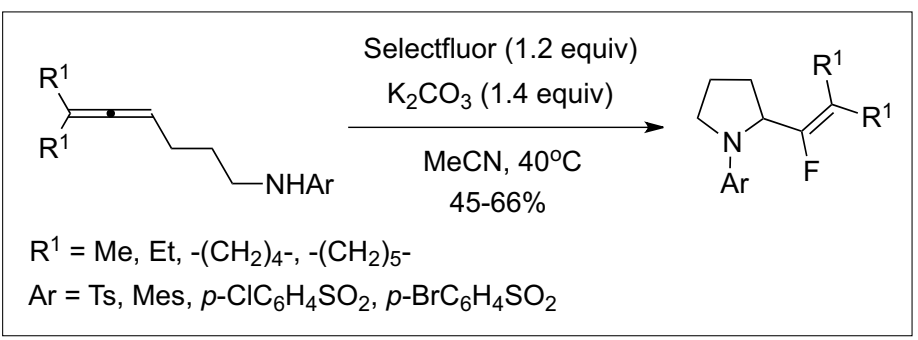

Scheme 4. Synthesis of pyrrolidine derivatives from $\gamma$-allenoic amides.

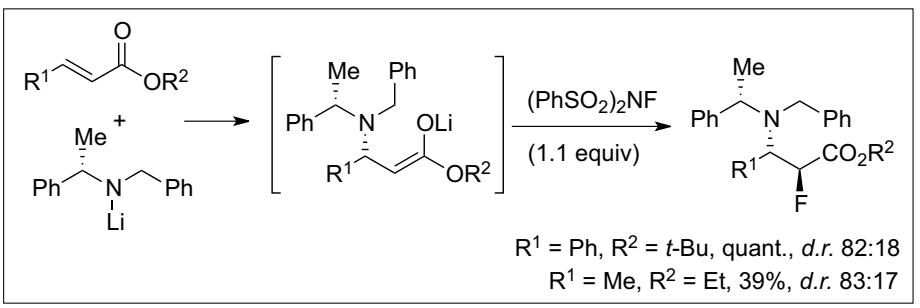

Scheme 5. Stereoselective synthesis of $\beta$-amino- $\alpha$-fluoro esters by fluorination of $\beta$-amino enolates.
1. a)

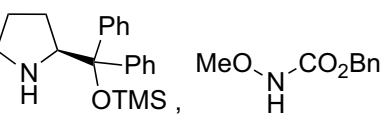

(20 mol\%)

(1.2 equiv)<smiles>[R]C=CC=O</smiles>

2. $\mathrm{NaBH}_{4}, \mathrm{MeOH}, 0^{\circ} \mathrm{C}$

$24-73 \%$

$\mathrm{R}=\mathrm{Et}, n-\mathrm{Pr}, i-\mathrm{Pr}, n-\mathrm{Bu}$, etc..

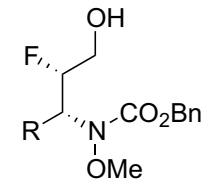

$98-99 \%$ ee b) NFSI ( 1 equiv), $0^{\circ} \mathrm{C}$

d.r. $=87: 13-98: 2$

Scheme 6. Synthesis of chiral $\alpha$-fluoro- $\beta$ amino alcohols.

\section{Nucleophilic Fluorination}

Fluorides have also been successfully employed in the aminofluorination of alkenes. A pioneering example of an alkene aminofluorination based on a nucleophilic source of fluorine was reported in 2003: cyclohexene reacted with 1-azido1,1,2,3,3-pentafluoropropane under harsh conditions to afford the desired transamino fluorinated adduct in addition to the product resulting from a competitive oxidative amination of the olefin in a $1: 1$ ratio (Scheme 9). ${ }^{[37]}$ The azide reacts with the double bond and upon $\mathrm{N}_{2}$ release, an aziridine is formed. Upon $\mathrm{F}^{-}$release, an $\alpha$-fluoroiminium cation is generated and trapped by the fluoride in a $S_{N} 2$ reaction. Final hydrolysis furnished the 1,2-transfluoroamide product.

Alternative strategies aiming at milder reaction conditions have mostly relied on 


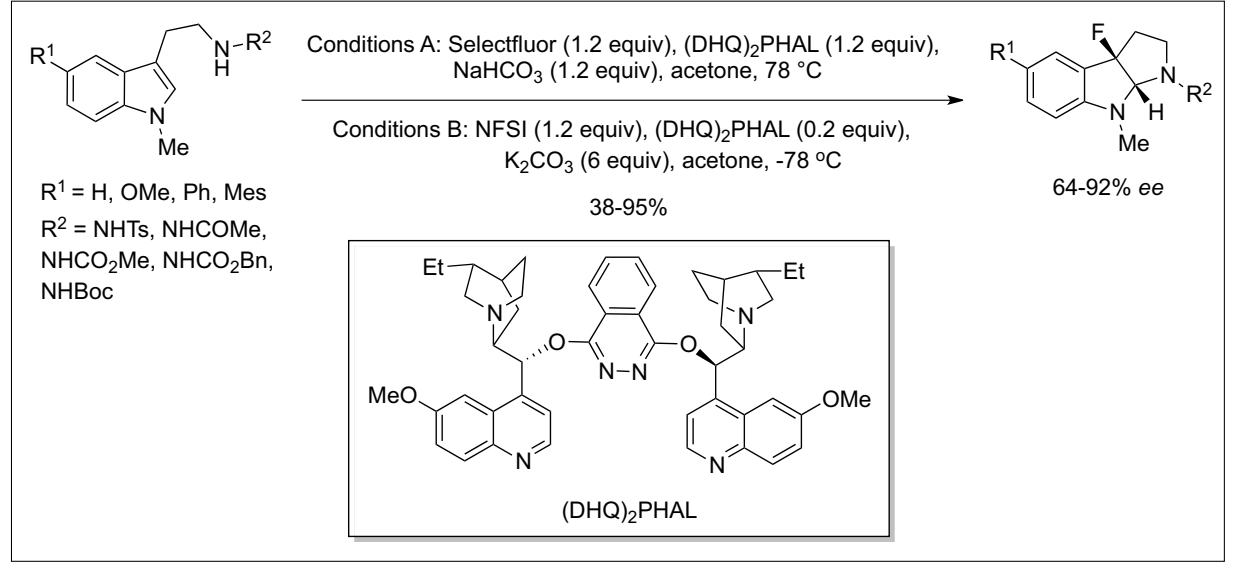

Scheme 7. Fluorocyclization of indoles. with the double bond was proposed in this case. The intramolecular nucleophilic attack of the amino group generates $\mathrm{F}^{-}$which traps in situ the cyclic carbocation intermediate produced in the preceding step. It is worth noting the dual role of $\mathrm{BF}_{3} \cdot \mathrm{Et}_{2} \mathrm{O}$ in these reactions, first as Lewis acid to activate iodosobenzene and also as the ultimate fluorine source.

An enantioselective version of these transformations was simultaneously reported by our group using a $(R, R)$-dimethyl lactate-based iodinium difluoride reagent. ${ }^{[40]}$ Fluoro piperidines were obtained in very good yields and excellent enantioselectivities upon crystallization. This method

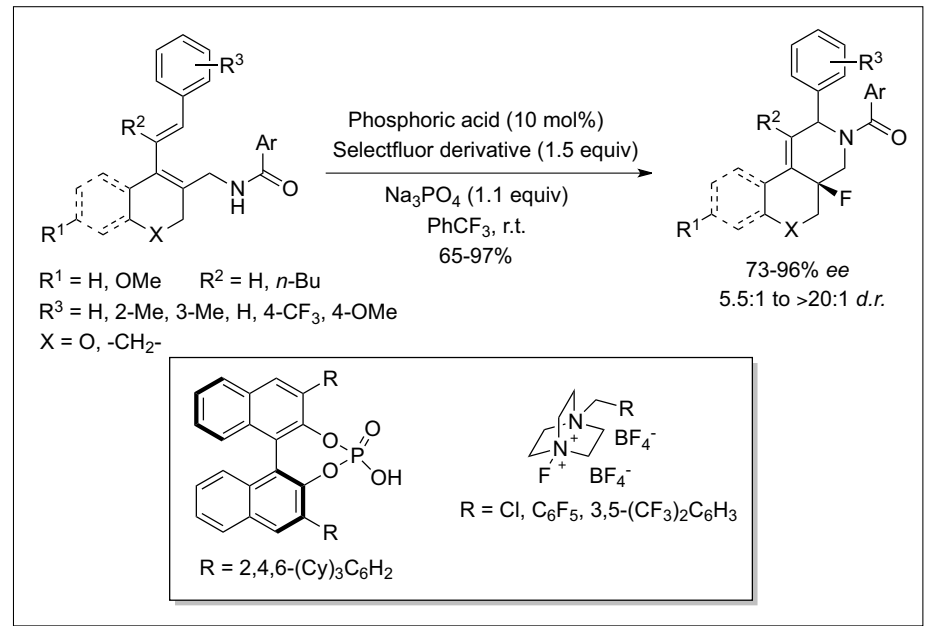

Scheme 8. Asymmetric 1,4-aminofluorination of conjugated dienes.

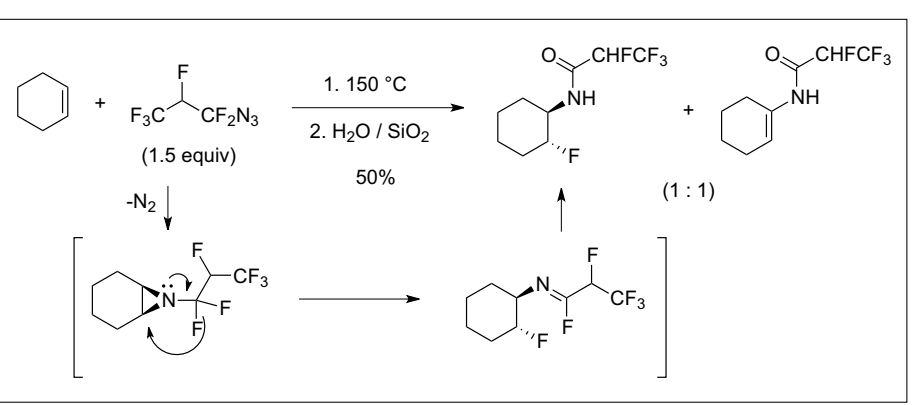

Scheme 9. Reaction of cyclohexene with 1-azido-1,1,2,3,3,3-hexafluoropropane.

the use of hypervalent iodine reagents. As such, an efficient metal-free intramolecular aminofluorination of $N$-tosyl pentenamines was reported using HF.Py as the fluorine source in the presence of $\mathrm{PhI}(\mathrm{OPiv})_{2}$ and $\mathrm{BF}_{3} \cdot \mathrm{Et}_{2} \mathrm{O}$ (Scheme 10). ${ }^{[38]}$ $\beta$-Fluoropiperidines were obtained with high regio- and stereoselectivity. The authors proposed a reaction between the sulfonamide nitrogen and the hypervalent iodine to generate an electrophilic iodonium intermediate which reacts with the double bond to generate carbocation A. Stabilization of this intermediate via neighboring group participation of the tosyl group could release a more stable intermediate B. Finally, fluoride is incorporated via $\mathrm{SN}_{2}$ reaction explaining the exclusive formation of the cis-products. In contrast, when the substrates bear a quaternary $\beta$-carbon, the axial alkyl group would prevent the formation of intermediate $\mathbf{B}$, producing a non-selective cis/trans mixture of products.

Interestingly, Zhang and co-workers disclosed an intramolecular aminofluorination reaction of homoallylic amines to obtain 3-fluoropyrrolidines in which $\mathrm{BF}_{3} \cdot \mathrm{Et}_{2} \mathrm{O}$, used in stoichiometric amount in combination with commercially available $\mathrm{PhIO}$, acted as the fluorine source (Scheme 11). ${ }^{[39]} \mathrm{A}$ mechanism involving the activation of $\mathrm{PhIO}$ by $\mathrm{BF}_{3} \cdot \mathrm{Et}_{2} \mathrm{O}$, followed by reaction of the activated iodo(III)-species could be nicely extended to the synthesis of $\beta$-fluorinated azepane derivatives, common scaffolds present in natural products as well as in synthetic bioactive molecules (Scheme 12). In the latter case though, a catalytic amount of cationic gold complexes was necessary to observe the desired reactivity, presumably to facilitate the activation of the olefin moiety. In addition, an intermolecular version of this methodology enabled the synthesis of unprecedented 2-fluoro-2-phenylethanamines (Scheme 13). This regioselective reaction nicely complements the previously electrophilic and palladium catalyzed aminofluorination methods (to be presented in section 5), which provide 2-fluoro-1-phenylethanamines as a result of the predominant carbocation stabilization at the benzylic position.

Based on control experiments and deu- 
terium labelling studies, the mechanism proposed for these transformations involve the oxidation of the sulphonamide group in the first step. The aminofluoro iodonium species then react with the olefin to give an aziridinium intermediate, which is attacked by fluoride onto the more substituted carbon atom to give the 6-endo cyclized products. Strong evidence supporting this mechanistic proposal was the isolation of an aziridinium intermediate in the aminofluorination reaction of 3-methylstyrene (Scheme 14).

The methods described in Schemes 10-14 utilize hypervalent iodine precursors and/or reagents in stoichiometric

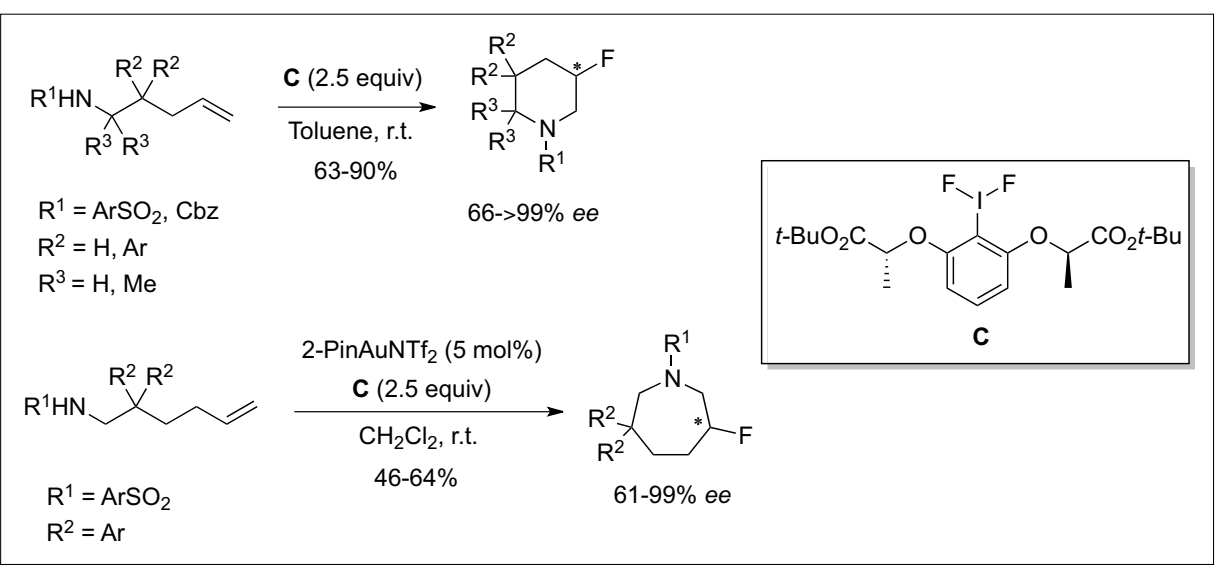

Scheme 12. Synthesis of fluoropiperidines and fluoroazepanes by intramolecular aminofluorination.

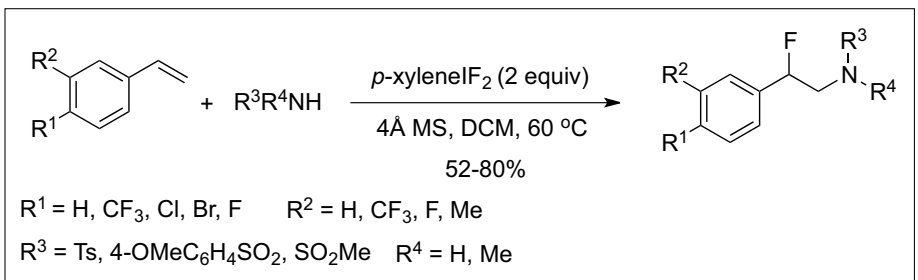

Scheme 13. Metal-free intermolecular aminofluorination.

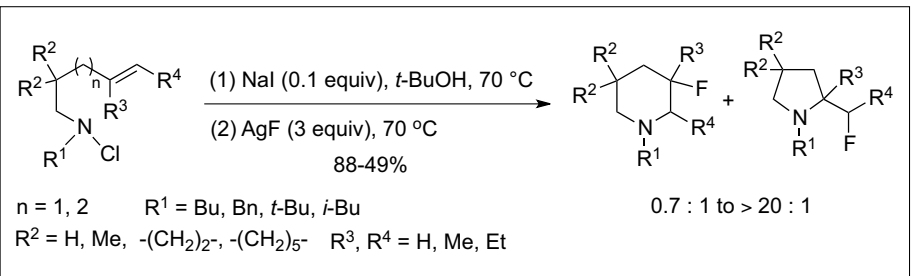

Scheme 15. Synthesis of azaheterocycles by aminofluorination of $\mathrm{N}$-chloramines.

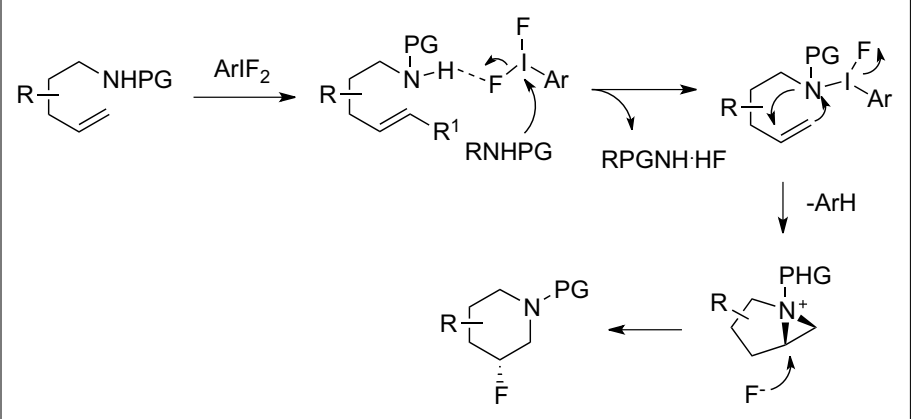

Scheme 14. Mechanistic proposal of the intramolecular aminofluorination of alkenes presented in Scheme 12.

fashion. However, catalytic versions of these processes, much more desirable from a synthetic point of view, have also been recently disclosed. Thus, Wang and co-workers partially overcame this limitation reporting an intramolecular aminofluorination of alkenes, which proceeded in the presence of a catalytic amount of $\mathrm{NaI}$ onto pre-oxidized $N$-chloramine substrates (Scheme 15). ${ }^{[41]}$ A similar mechanism involving an aziridinium intermediate was proposed upon in situ generation of an $N$-iodoamine intermediate. An alternative catalytic strategy for the aminofluorination of $\omega$-aminoalkenes was described using the system iodoarene/MCPBA/HF.Py (Scheme 16). ${ }^{[42]}$ Hypervalent iodine species were generated in situ by oxidation of the iodoarene with MCPBA followed by reaction with $\mathrm{HF}$ to give $\mathrm{ArIF}_{2}$. The reaction then seems to follow a mechanism related to that shown in Scheme 14.

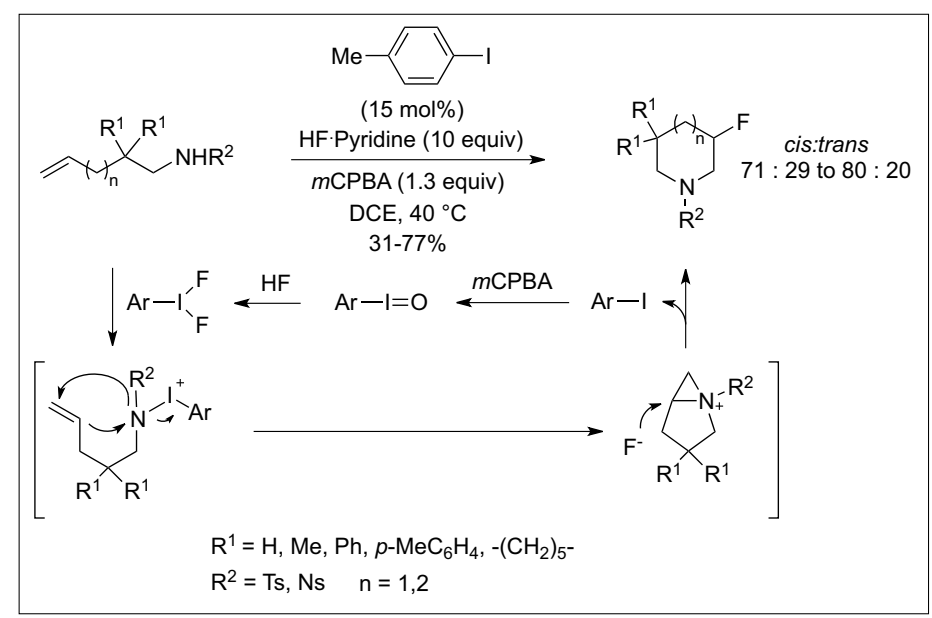

Scheme 16. lodoarene catalyzed aminofluorination by Ar-I/HF-pyridine/ $m$ CPBA.

\section{Radical Fluorination}

Although few examples have been reported, the in situ generation of fluorine radicals offers a complementary reactivity to the electrophilic and nucleophilic methods described in the previous sections. $\mathrm{Li}$ and co-workers reported the synthesis of 5-fluoromethyl-substituted $\gamma$-lactams through an intramolecular radical aminofluorination of $\mathrm{N}$-arylpent-4-enamides in aqueous media. $\mathrm{AgNO}_{3}$ was used as catalyst and Selectfluor seems to act both as the oxidant and fluorine donor in these transformations. $\mathrm{A} \mathrm{Ag}(\mathrm{III}) \mathrm{F}$ intermediate seems to be generated in situ from the interaction of $\mathrm{AgNO}_{3}$ with Selectfluor. The enamide substrate can be oxidized by this complex to generate $\operatorname{Ag}(\mathrm{II}) \mathrm{F}$ and an arene radical cation, which undergoes proton abstraction to give an amidyl radical. A 5-exo intramolecular addition onto the alkene generates a carbon-centered radical which is fluorinated with $\mathrm{Ag}(\mathrm{II}) \mathrm{F}$ to produce the fluorinated lactams regenerating the $\operatorname{Ag}(\mathrm{I})$ species to close the catalytic cycle (Scheme 17). ${ }^{[43]}$

In a related process, unactivated alkenes could be transformed into $\beta$-fluorinated alkyl azides through a metal-free reaction involving $\mathrm{TMSN}_{3}$ and Selectfluor in aqueous acetonitrile at room temperature (Scheme 18). ${ }^{[44]}$ The azido group is added to the less sterically hindered carbon of the carbon-carbon double bond in a highly regioselective process which showed wide functional group compatibility and good stereoselectivity. 


\section{Transition Metal-catalyzed Oxidative Fluorination}

Transition metals have been extensively used in order to achieve milder reaction conditions for the construction of $\mathrm{C}-\mathrm{F}$ bonds. Taking advantage of their accused electrophilic nature and thus higher affinity for the highly solvated, thus poorly nucleophilic $\mathrm{F}^{-}$ion, transition metals in a high oxidation state can facilitate the formation of $\mathrm{C}-\mathrm{F}$ bonds via reductive elimination or, in those in a lower oxidation state, via $\mathrm{S}_{\mathrm{N}} 2$ reactions. A pioneering example of a metal-catalyzed aminofluorination of alkenes was reported by Liu and co-workers in 2009. Palladium catalyzed a 6-endo-selective intramolecular aminofluorination of unsaturated $\mathrm{N}$-sulfonamides using $\mathrm{AgF}$ as the fluorine donor in the presence of $\mathrm{PhI}(\mathrm{OPiv})_{2}$ as stoichiometric oxidant (Scheme 19). ${ }^{[45]}$ Upon trans-

$$
\begin{aligned}
& \mathrm{R}=\mathrm{H}, \mathrm{Me}_{2},-\left(\mathrm{CH}_{2}\right)_{4^{-}}, \\
& -\left(\mathrm{CH}_{2}\right)_{5^{-}}, \mathrm{Me}, \mathrm{Ph} \\
& \mathrm{X}=\mathrm{C}, \mathrm{O}, \mathrm{N}
\end{aligned}
$$

Proposed mechanism:

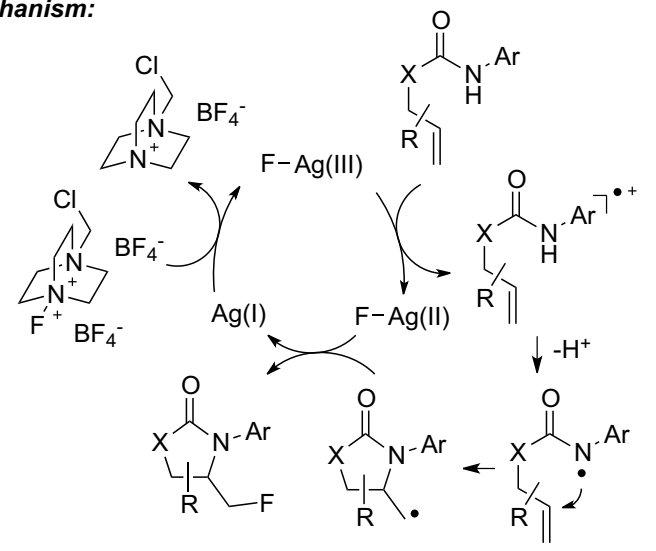

Scheme 17. Silvercatalyzed aminofluorination of unactivated alkenes.

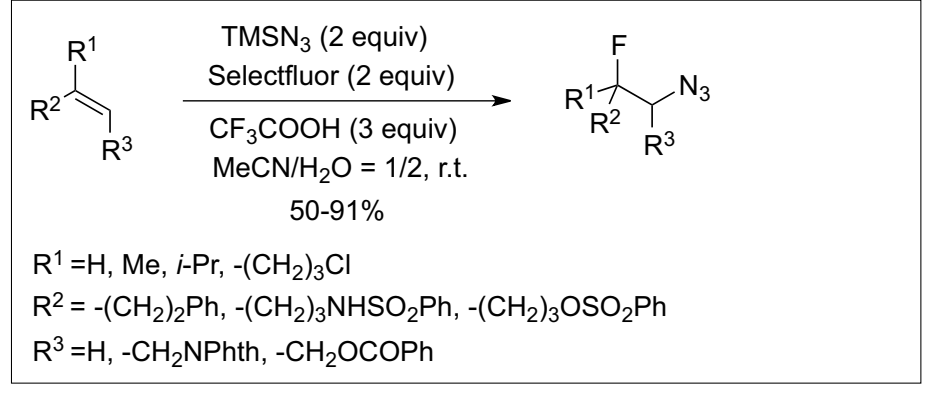

Scheme 18. Azidofluorination of alkenes.

amino palladation of the double bond, the formation of a $\mathrm{Pd}(\mathrm{II})$ intermediate is proposed. Oxidation with $\mathrm{PhI}(\mathrm{OPiv})_{2}$, delivers $\mathrm{Pd}(\mathrm{IV})-\mathrm{F}$ species, which undergo reductive elimination to deliver the $\mathrm{Csp}^{3}-\mathrm{F}$ bond. Alternatively, a competitive $\mathrm{S}_{\mathrm{N}} 2$ type mechanism operating on $\mathrm{Pd}(\mathrm{II})$ intermediates cannot be ruled out. Interestingly, 5-exo-cyclizations seem to be favored under similar reaction conditions when unsaturated amides are used as the nitrogenprotecting group. Thus, 2-(fluoromethyl) pyrrolidines and imidazolidinones were selectively obtained with this protocol when hexafluoroisopropyl alcohol was used as additive (Scheme 20). ${ }^{[46]}$

A palladium-catalyzed intermolecular aminofluorination of activated styrene substrates was developed by the same group with NFSI as the source of both nitrogen and fluorine. ${ }^{[47]}$ Both $\mathrm{Pd}(0) / \mathrm{Pd}(\mathrm{II})$ and $\mathrm{Pd}(\mathrm{II}) / \mathrm{Pd}(\mathrm{IV})$ catalytic cycles can be proposed as plausible mechanisms in this transformation. It is worth noting that both catalytic cycles involve fluoropalladation of styrenes as a key step to construct $\mathrm{C}-\mathrm{F}$ bonds while the presence of bidentate nitrogen ligand is necessary for a successful reaction outcome (Scheme 21). Similar conditions were used for an intramolecular version of this reaction. ${ }^{[48]}$

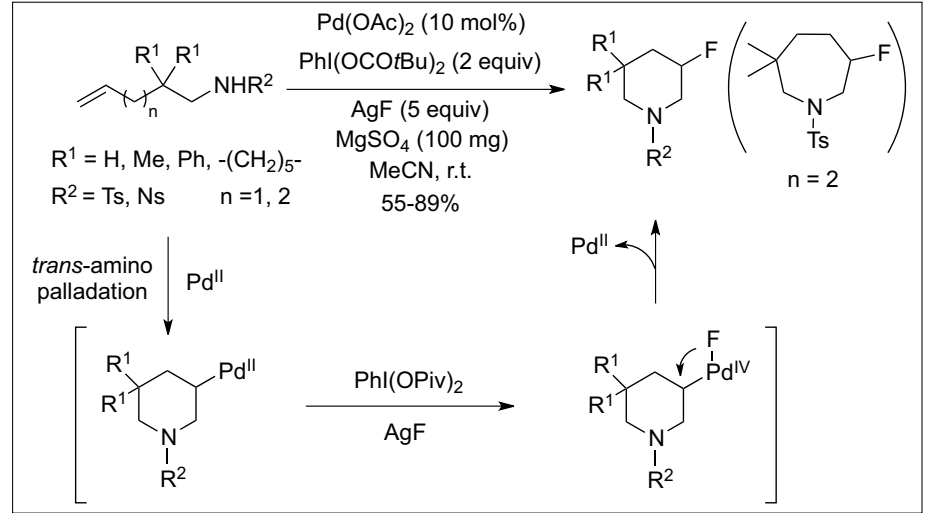

Scheme 19. Palladium-catalyzed intramolecular aminofluorination of unactivated alkenes.

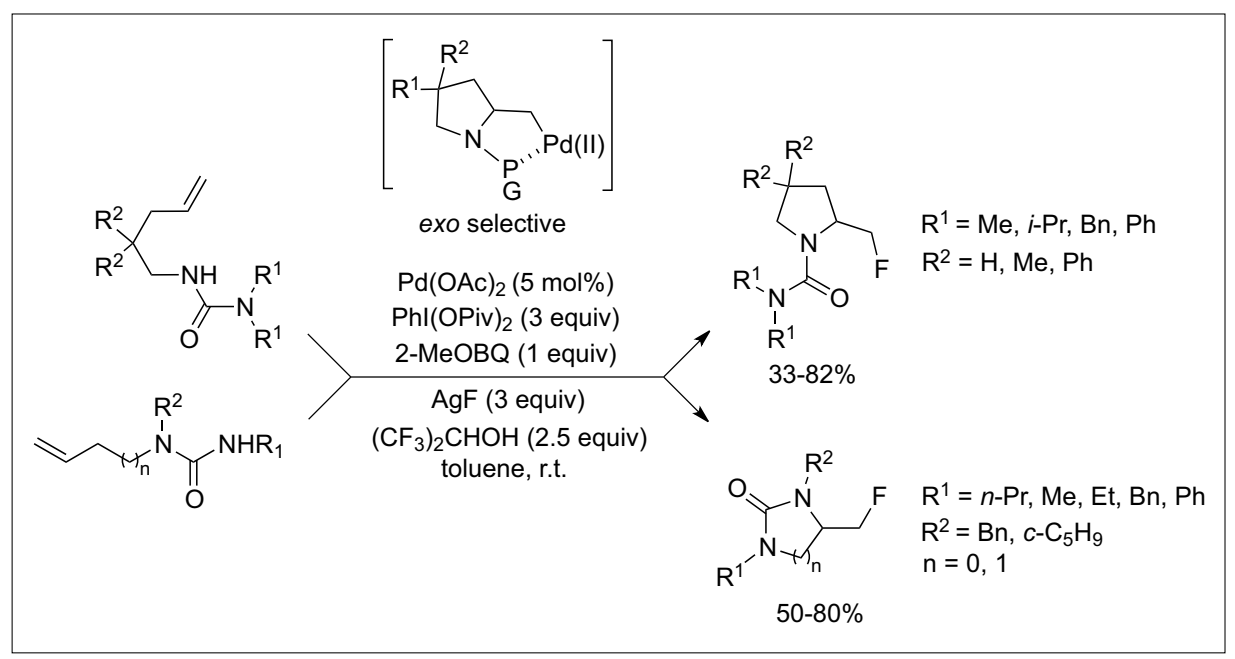

Scheme 20. Pd-catalyzed aminofluorination of alkenes.

The methodologies presented above could be extended to allenes providing a variety of 4-fluoro-dihydropyrrole derivatives in moderate to excellent yields in the presence of silver as catalyst (Scheme 22). ${ }^{[49]}$ The authors proposed a mechanism involving a $\mathrm{Ag}(\mathrm{I}) / \mathrm{Ag}(\mathrm{II})$ catalytic cycle, in which the vinyl $\mathrm{C}-\mathrm{Ag}$ bond is cleaved using $\mathrm{N}$-fluorobenzenesulfonimide to form the vinyl $\mathrm{C}-\mathrm{F}$ bonds. In addition, the obtained 4-fluoro-dihydropyrroles could be further converted to the corresponding 4-fluoropyrroles in a one-pot fashion in good yields. 


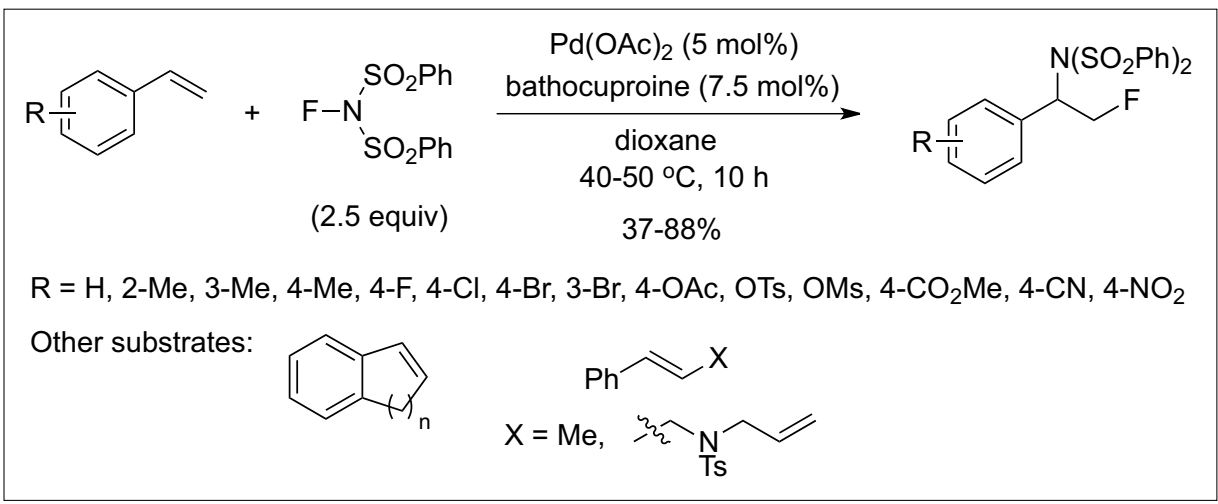

Scheme 21. Palladium-catalyzed intermolecular aminofluorination of styrenes.

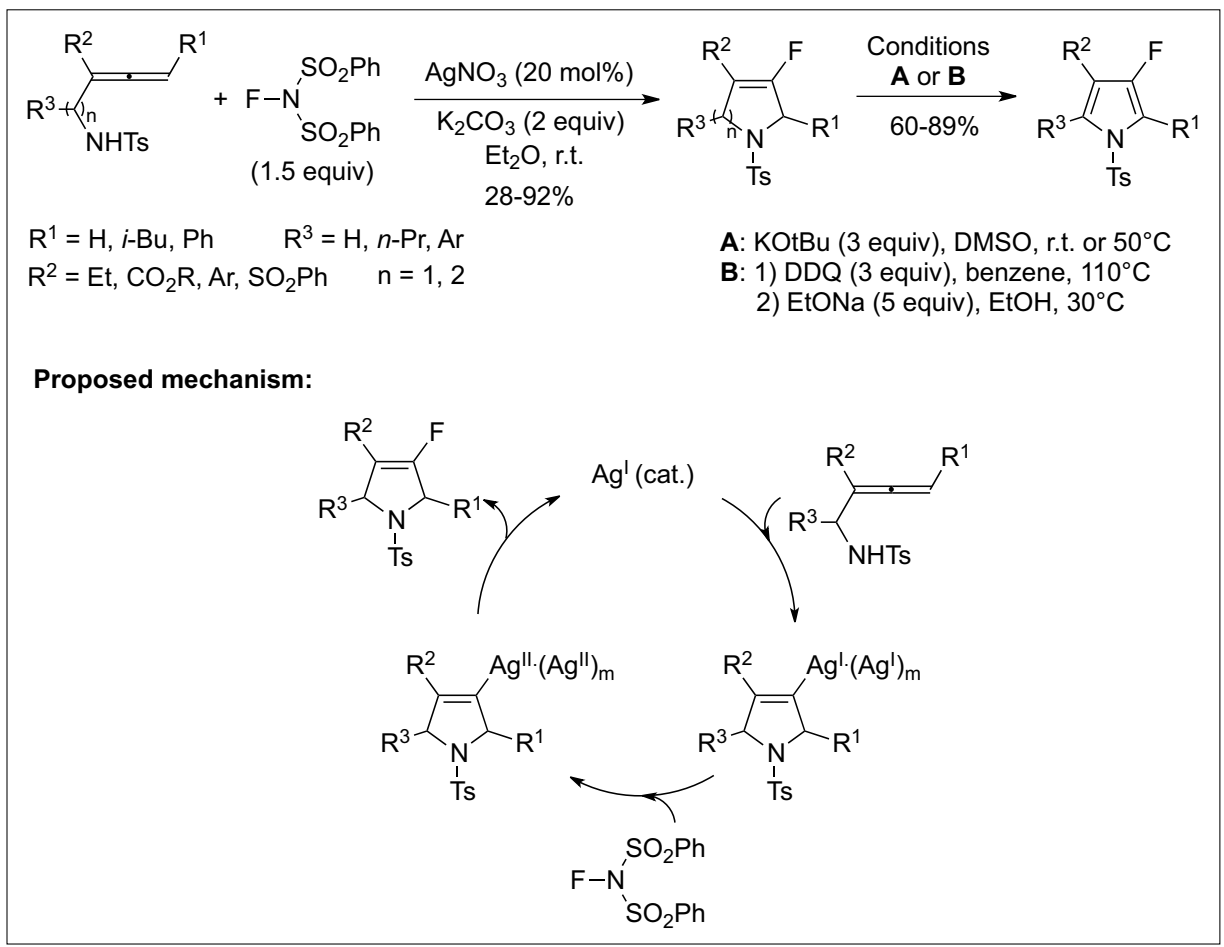

Scheme 22. Intramolecular aminofluorination of activated allenes.

\section{Conclusions}

Considerable progress in the development of alkene aminofluorination reactions has been achieved in recent years enabling unprecedented levels of chemo-, regio-, and stereoselectivity. The diversity of reaction mechanisms and protocols applied to the synthesis of $\beta$-aminofluorinated compounds grant further applications of these methodologies in more complex settings. Improvements on the substrate scope, atom economy and degree of absolute stereocontrol in these transformations will certainly trigger new developments in the field in the near future.

\section{Acknowledgements}

Financial support from the University of Zürich and the European Union through an ERC Starting Grant (no. 307948) is kindly acknowledged.
[14] R. Zhu, S. L. Buchwald, Angew. Chem. Int. Ed. 2012, 51, 1926.

[15] L. Zhu, H. Yu, Z. Xu, X. Jiang, L. Lin, R. Wang, Org. Lett. 2014, 16, 1562.

[16] H. Wang, Y. Wang, D. Liang, L. Liu, J. Zhang, Q. Zhu, Angew. Chem. Int. Ed. 2011, 50, 5678.

[17] P. H. Fuller, J.-W. Kim, S. R. Chemler, J. Am. Chem. Soc. 2008, 130, 17638.

[18] T. de Haro, C. Nevado, Angew. Chem. Int. Ed. 2011, 50, 906

[19] E. J. Alexanian, C. Lee, E. J. Sorensen, J. Am. Chem. Soc. 2005, 127, 7690.

[20] D. T. Smith, J. T. Njardarson, Angew. Chem. Int. Ed. 2014, 53, 4278 .

[21] C. Röben, J. A. Souto, Y. González, A. Lishchynskyi, K. Muñiz, Angew. Chem. Int. Ed. 2011, 50, 9478.

[22] E. L. Ingalls, P. A. Sibbald, W. Kaminsky, F. E. Michael, J. Am. Chem. Soc. 2013, 135, 8854.

[23] B. Han, X.-L. Yang, R. Fang, W. Yu, C. Wang, X.-Y. Duan, S. Liu, Angew. Chem. Int. Ed. 2012, 51, 8816.

[24] P. Chavez, J. Kirsch, C. H. Hovelmann, J. Streuff, M. Martinez-Belmonte, E. C. EscuderoAdan, E. Martin, K. Muniz, Chem. Sci. 2012, 3, 2375.

[25] X. Ji, H. Huang, W. Wu, H. Jiang, J. Am. Chem. Soc. 2013, 135, 5286

[26] M. T. Bovino, S. R. Chemler, Angew. Chem. Int. Ed. 2012, 51, 3923.

[27] E. Merino, C. Nevado, Chem. Soc. Rev. 2014, doi: 10.1039/C4CS000025K.

[28] S. Stavber, T. S. Pecan, M. Papez, M. Zupan, Chem. Commun. 1996, 2247.

[29] S. Stavber, T. Sotler Pecan, M. Zupan, J. Chem. Soc., Perkin Trans. 2 2000, 1141.

[30] J. S. Yadav, B. V. Subba Reddy, D. Narasimha Chary, D. Chandrakanth, Tetrahedron Lett. 2009, 50, 1136.

[31] N. Shibata, T. Tarui, Y. Doi, K. L. Kirk, Angew. Chem. Int. Ed. 2001, 40, 4461.

[32] H. Cui, Z. Chai, G. Zhao, S. Zhu, Chin. J. Chem. 2009, 27, 189.

[33] P. C. Andrews, V. Bhaskar, K. M. Bromfield, A. M. Dodd, P. J. Duggan, S. A. M. Duggan, T. D. McCarthy, Synlett 2004, 791.

[34] C. Appayee, S. E. Brenner-Moyer, Org. Lett. 2010, 12, 3356.

[35] O. Lozano, G. Blessley, T. Martinez del Campo, A. L. Thompson, G. T. Giuffredi, M. Bettati, M. Walker, R. Borman, V. Gouverneur, Angew. Chem. Int. Ed. 2011, 50, 8105.

[36] H. P. Shunatona, N. Früh, Y.-M. Wang, V. Rauniyar, F. D. Toste, Angew. Chem. Int. Ed. 2013, 52, 7724.

[37] S. A. Lermontov, A. N. Pushin, S. V. Shkavrov, A. G. Polivanova, Russ. Chem. Bull. 2010, 59, 2114

[38] Q. Wang, W. Zhong, X. Wei, M. Ning, X. Meng, Z. Li, Org. Biomol. Chem. 2012, 10, 8566.

[39] J. Cui, Q. Jia, R.-Z. Feng, S.-S. Liu, T. He, C. Zhang, Org. Lett. 2014, 16, 1442.

[40] W. Kong, P. Feige, T. de Haro, C. Nevado, Angew. Chem. Int. Ed. 2013, 52, 2469.

[41] H.-T. Huang, T. C. Lacy, B. Błachut, G. X. Ortiz, Q. Wang, Org. Lett. 2013, 15, 1818.

[42] S. Suzuki, T. Kamo, K. Fukushi, T. Hiramatsu, E. Tokunaga, T. Dohi, Y. Kita, N. Shibata, Chem. Sci. 2014, DOI: 10.1039/C3SC53107D.

[43] Z. Li, L. Song, C. Li, J. Am. Chem. Soc. 2013, 135, 4640.

[44] Z. Li, C. Zhang, L. Zhu, C. Liu, C. Li, Org. Chem. Front. 2014, 1, 100

[45] T. Wu, G. Yin, G. Liu, J. Am. Chem. Soc. 2009, 131, 16354.

[46] T. Wu, J. Cheng, P. Chen, G. Liu, Chem. Commun. 2013, 49, 8707.

[47] S. Qiu, T. Xu, J. Zhou, Y. Guo, G. Liu, J. Am. Chem. Soc. 2010, 132, 2856.

[48] T. Xu, S. Qiu, G. Liu, Chin. J. Chem. 2011, 29, 2785.

[49] T. Xu, X. Mu, H. Peng, G. Liu, Angew. Chem. Int. Ed. 2011, 50, 8176 .
Int. Ed. 2010, 49, 4491.
[13] A. Wang, H. Jiang, H. Chen, J. Am. Chem. Soc. 2009, 131,3846 . 